\title{
[gw22-e0732] ADIPONECTIN THROUGH ITS BIPHASIC SERUM LEVEL IS A USEFUL BIOMARKER DURING TRANSITION FROM DIASTOLIC DYSFUNCTION TO SYSTOLIC DYSFUNCTION
}

Jingmin Zhou, Mingqiang Fu, Aijun Sun, Xuejuan Jin, Chunlin Zhong, ShiJun Wang, Michael Fu, Yunzeng Zou, Junbo Ge Department of Cardiology, Shanghai Cardiovascular Institute, Zhongshan Hospital, Fudan University, Shanghai, China

10.1136/heartjnl-2011-300867.729

Aims To examine whether adiponectin is associated with disease progression of heart failure from hypertension in rats in comparison with other known biomarkers and echocardiographic parameters.

Methods Spontaneously hypertensive rats (SHR, $\mathrm{n}=35$ ), aged 1 month, were used and followed up to 18 months. High frequency echocardiography was performed both at baseline and every 3 months thereafter. Moreover, serum levels of $\mathrm{N}$-terminal pro-natriuretic peptide (NT-proBNP) and interleukin 6 (IL- 6 ) as well as serum level and tissue expression of adiponectin were measured in the same time as echocardiography.

Results Our results clearly demonstrated time-dependent progression of hypertension and heart dysfunction as evidenced by gradually increased left ventricular mass index, NT-proBNP, IL- 6 and gradually decreased cardiac function as assessed by echocardiography. Meanwhile, tissue and serum adiponectin decreased from 3 months and reached plateau until 12 month in parallel with decreasing of cardiac diastolic function and then kept increasing prior to occurrence of systolic dysfunction. And adiponectin concentration is inversely related with NT-proBNP, IL- 6 and E/E' (correlation coefficient $(r)=-0.756$ for NT-proBNP, $p<0.001,-0.635$ for IL- $6, p=0.002$ and -0.626 for $E / E^{\prime}, p=0.002$, respectively) while positively correlated with $E / A$ and $E^{\prime} / A^{\prime}$ ( $r=$ was 0.683 for $E / A, p=0.001$, 0.671 for $E^{\prime} / A^{\prime}, p=0.001$, respectively). No difference for adiponectin distribution among visceral adipose tissues was found.

Conclusions Adiponectin through its biphasic serum level is a useful biomarker during transition from diastolic dysfunction to systolic dysfunction. 